\title{
APPLICATION OF LATTICE BOLTZMANN MODEL TO SIMULATE DIFFERENT TEST CASES IN TWO DIMENSIONAL ENCLOSURE
}

\author{
Sagnik Banik \\ Student of Aerospace \\ Engineering,IIEST,Howrah,West Bengal Bengal
}

ASTRACT - Lattice Boltzmann Method have been advantageous in simulating complex boundary conditions and solving for fluid flow parameters by streaming and collision processes.This paper includes the study of three different test cases in a confined domain using the method of the Lattice Boltzmann model. 1. An SRT (Single Relaxation Time) approach in the Lattice Boltzmann model is used to simulate Lid Driven Cavity flow for different Reynolds Number (100, 400 and 1000) with a moment-based boundary condition is used for more accurate results. 2. A Thermal Lattice BGK (Bhatnagar-Gross-Krook) Model is developed for the Rayleigh Benard convection for both test cases - Horizontal and Vertical Temperature difference, considered separately for a Boussinesq incompressible fluid with different Rayleigh number. 3.The phase change problem governed by the heat-conduction equation is studied using the enthalpy based Lattice Boltzmann Model to provide a better understanding of the heat transport phenomenon.An approximate velocity scale is chosen to ensure that the simulations are within the incompressible regime.The simulated results demonstrate excellent agreement with the existing benchmark solution implicates the viability of this method for complex fluid flow problems.

KEYWORDS: Boussinesq, LBM, Nusselt, Prandtl, Rayleigh,SRT, Stefan

\section{INTRODUCTION}

For the equations governing the fluid mechanics, analytical solution exists for only few basic cases.With more complexity in geometry and boundary condition numerical methods become an absolute necessity.The Lattice Boltzmann Method originally derived from the lattice gas cellular

\author{
Prof Jean Yves Trepaier \\ Professor and Directory of \\ Experts,Polytechnique Montreal,Quebec,Canada
}

automaton models has an upper hand due to its robust implementation and ease to parallelise. The extensive studies of this method including Succi(2001), WolfGladrow(2000), Rothman and Zaleski(1997), Chen and Doolen(1998) laid the mathematical foundation of this method.This method has been successful in simulating some of the most complex flows including those discussed in this paper.The lid driven cavity flow studied by Ghia [Ghia et al.(1982)Ghia, Ghia, and Shin] is a classic benchmark problem for assessing the accuracy of any algorithm. A moment based boundary condition [Bennett(2010)] is implemented on the population distribution of LBM.The rayleigh benard problem of natural convection has been of great interest due its wide range of applications in heat transport phenomenon. The bouoyancy driven fluid flow with adibatic top and bottom walls representing the horizontal temperature difference case of rayleigh benard problem has been a topic of extensive study Wang et al.(2013), Kao and Yang et al(2007), Guo et al.(2002) including the classic benchmark solution of deVahlDavis et al(1983). The vertical case has been also mentioned in several literatures(ref).The melting and solidifiacation process has also be sucessfully implemented through the enthalpy based thermal lattice boltzmann model Jiaung et al.(2001), Chakraborty and Chatterjee et al(2007), Huber et al.(2008), Huang and Wu et al(2015), Huo and Rao et al(2017), Chatterjee et al(2010)].

We investigated the applicability of LBM for the three aforementioned cases by comparing the calculated governing parameters with the existing benchmark solutions. The lid driven cavity flow is studied for different Reynolds number and aspect ratio of cavity.The rayleigh benard problem is implemented with a definite forcing scheme and stability criteria and the phase change problem is simulated through the enthalphy based model and the resulting temperature distribution is compared with the avalaible analytical solution Jiaung et al.(2001). 


\section{International Journal of Engineering Applied Sciences and Technology, 2019 \\ Vol. 4, Issue 8, ISSN No. 2455-2143, Pages 14-26 \\ Published Online December 2019 in IJEAST (http://www.ijeast.com)}

\section{NUMERICAL METHODOLOGY}

The fundamental idea is that fluids can be imagined as consisting of large number of small particles moving with random motions. The exchange of momentum and energy is achieved through particle streaming and collision. This process can be modeled by the Boltzmann transport equation which is

$\frac{\partial f}{\partial t}+u \Delta f=\Omega$

where $\mathrm{f}$ is the particle distribution, $\mathrm{u}$ is the particle velocity and $\Omega$ is the collision operator given by $\Omega=$ $-\frac{f_{i}-f_{i}^{e q}}{\tau} \Delta t$. The relaxation time $\tau$ can be derived from the governing parameters depending upon the macroscopic parameter $f$ represents.

For a two dimensional model the number of particle is restricted to stream of 5 or 9 directions including one staying at rest at the center giving the D2Q5 and D2Q9 model.Each model has its own set of distribution functions and weights.For the D2Q9 model ,each particle on the lattice we associate a discrete probability distribution function $f_{i}(x, t)$ for $\mathrm{i}=0 \ldots . .8$, which describes the probability of the streaming in one particular direction.
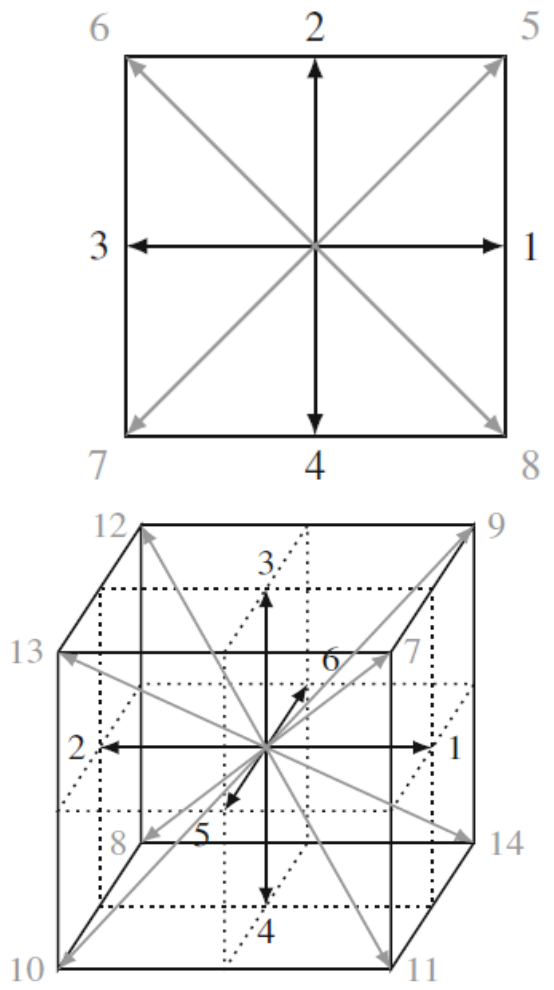

Figure 1:Representation of the model D2Q9(left) and D3Q15(right) used in LBM
The macroscopic fluid density is given as the sum of this particle distribution functions.

$\rho(x, t)=\Sigma_{n=0}^{8} f_{i}(x, t)$

Accordingly the macroscopic velocity $u$ is an average of the velocities weighted by the distribution functions $f_{i}$,

$\rho u=\Sigma_{n=0}^{8} f_{i}(x, t) e_{i}$

where $e_{i}$ is the discrete velocity of each of the particle distribution. The discrete velocity distribution along the different directions of a $\mathrm{D} 2 \mathrm{Q} 9$ model is given by

$\left(\begin{array}{ll}(0,0) & i=0 \\ c\left(\cos \left[(i-1) \frac{\pi}{2}\right], \sin \left[(i-1) \frac{\pi}{2}\right]\right) & i=1,2,3,4, \\ \sqrt{2} c\left(\cos \left[(2 i-1) \frac{\pi}{4}\right], \sin \left[(2 i-1) \frac{\pi}{4}\right]\right) & i=5,6,7,8,\end{array}\right.$ (4) where $c=\frac{\Delta x}{\Delta t}$ is the lattice speed of the domain.

The key steps in the LBM are the streaming and the coliision process which are given by

$f_{i}\left(x+e_{i} \Delta t, t+\Delta t\right)-f(x, t)=-\frac{f_{i}(x, t)-f_{i}^{e q}(x, t)}{\tau}$

where $\tau$ is the relaxation time of the distribution and $f_{i}^{e q}$ is the equilibrium distribution function.Using the Bhatnagar-Gross-Krook(BGK) collision approximation the equilibrium distribution function for $\mathrm{D} 2 \mathrm{Q} 9$ is given by

$$
f_{i}^{e q}(x, t)=w_{i} \rho\left(1+\frac{e_{i} u}{c_{s}^{2}}+\frac{\left(e_{i} u\right)^{2}}{2 c_{s}^{4}}-\frac{u \cdot u}{2 c_{s}^{2}}\right)
$$

and the weights are given by

$$
w_{i}=\left(\begin{array}{ll}
4 / 9 & \mathrm{i}=0 \\
1 / 9 & \mathrm{i}=1,2,3,4 \\
1 / 18 & \mathrm{i}=5,6,7,8
\end{array}\right.
$$

where $c_{s}$ represents the speed of sound in lattice. Parameters governing the fluid flow show dependence on the lattice units. The test cases discussed in the subsequent section has unique boundary condition each carefully implemented on the particle distribution.

\section{THE LID CAVITY PROBLEM}

The Lid Driven Cavity flow is a classic benchmark problem studied extensively using the LGBK approach and proved to provide stable solution when compared with the Naiver-Stokes solution of Ghia [Ghia et al.(1982)].Researches on the Lid Driven Cavity have investigated on the various effects of the change in aspect ratio, the influence of the critical Reynolds number and effects of its change within the two dimensional cavity. The LBM method mentioned here focuses on the aspects of the Lid Driven Cavity 
that have a direct influence with the change in the Reynolds Number.We have adopted a SRT approach which provided a convincing result with the existing solution. The aspect ratio for the cavity is given by $A R=\frac{W}{H}$ where $\mathrm{W}$ is the width of the cavity and $\mathrm{H}$ is the depth.

\subsection{Numerical Methodology:Single Population}

\section{Lattice Bolltzmann SRT Model}

We have adopted a single population D2Q9 model for this test case for which the streaming and the collision steps is given by eq(5).The fluid kinematic viscosity is related to the relaxation time in D2Q9 model by $v=\frac{2 \tau-1}{6} \frac{(\Delta x)^{2}}{\Delta t}$. A simple bounce back condition is applied at all the boundaries except the top i.e the lid where the velocity is imparted.The simple bounce back condition simulate for the rigid boundaries of the domain with no penetration and velocity.A carefully devised hydrodynamic moment based boundary conditions given by Bennett [Bennett(2010)] are used for the top boundary

$\rho u_{x}=\Sigma f_{i} e_{i x}=f_{1}-f_{3}+f_{5}-f_{6}-f_{7}+f_{8}$

$\rho u_{y}=\Sigma f_{i} e_{i y}=f_{2}-f_{4}+f_{5}+f_{6}-f_{7}-f_{8}$

$\Pi_{x x}=\Sigma f_{i} e_{i x}^{2}=f_{1}+f_{3}+f_{5}+f_{6}+f_{7}+f_{8}$

$\Pi_{y y}=\Sigma f_{i} e_{i y}^{2}=f_{2}+f_{4}+f_{5}+f_{6}+f_{7}+f_{8}$

$\Pi_{x y}=\Sigma f_{i} e_{i x} e_{i y}=f_{5}-f_{6}+f_{7}-f_{8}$

We use the first three moments and impose a no slip and o flux boundary condition

$$
\rho u_{x}=\rho u ; \rho u_{x}=0 ; \Pi_{x x}=\rho / 3+\rho U^{2}
$$

where $(\mathrm{U}, 0)$ is the velocity applied on the top boundary. By solving we get the unknown distributions at the top boundary

$$
\begin{gathered}
\rho=f_{0}+f_{1}+f_{3}+2\left(f_{2}+f_{5}+f_{6}\right) \\
f_{4}=f_{1}+f_{3}+f_{2}+2\left(f_{5}+f_{6}\right)-\frac{\rho}{3}-\rho U^{2} \\
f_{7}=\frac{\rho}{6}+\frac{\rho U^{2}}{2}-\frac{\rho U}{2}-f_{3}-f_{6} \\
f_{8}=\frac{\rho}{6}+\frac{\rho U^{2}}{2}+\frac{\rho U}{2}-f_{1}-f_{5}
\end{gathered}
$$

For the nodes at the corner we have five unknown distributions.For this we need all the moments together with a condition on the shear stress $\Pi_{x y}$ set to zero.We get for the north(top)west corner

$$
\begin{aligned}
& f_{1}=\frac{2 \rho}{3}-f_{0}-f_{3} \\
& f_{4}=\frac{2 \rho}{3}-f_{0}-f_{2} \\
& f_{5}=\frac{\rho}{6}-f_{2}-f_{6} \\
& f_{7}=\frac{\rho}{6}-f_{3}-f_{6} \\
& f_{8}=-\frac{2 \rho}{6}+f_{0}+f_{2}+f_{3}+f_{6}
\end{aligned}
$$

with $\rho=f_{0}+2 f_{3}+4 f_{6}+2 f_{2}$. For the other corners the similar approach is taken to determine the unknown distribution.

\subsection{Results}

For this simulation a domain of $\mathrm{AR}=1$ is adopted with a meshing grid size of $\Delta x=\Delta y=1$ with $\Delta t=1$. Three different Reynolds Number i.e 100,400 and 1000 are studied and quantities of interest such as the horizontal and vertical velocities at the centre of the domain are compared with the benchmark results Ghia et al.(1982) given in Fig. 2, Fig. 3 and Fig. 5.The contours of horizontal velocity for two cases of $\mathrm{Re}=100$ and $\mathrm{Re}=1000$ are given in Fig. 6. With he increase in Reynolds number the center of the circulation shifts more towards the center.In addition we investigated for $A R=4$ representing the shallow cavity case Fig. 4.
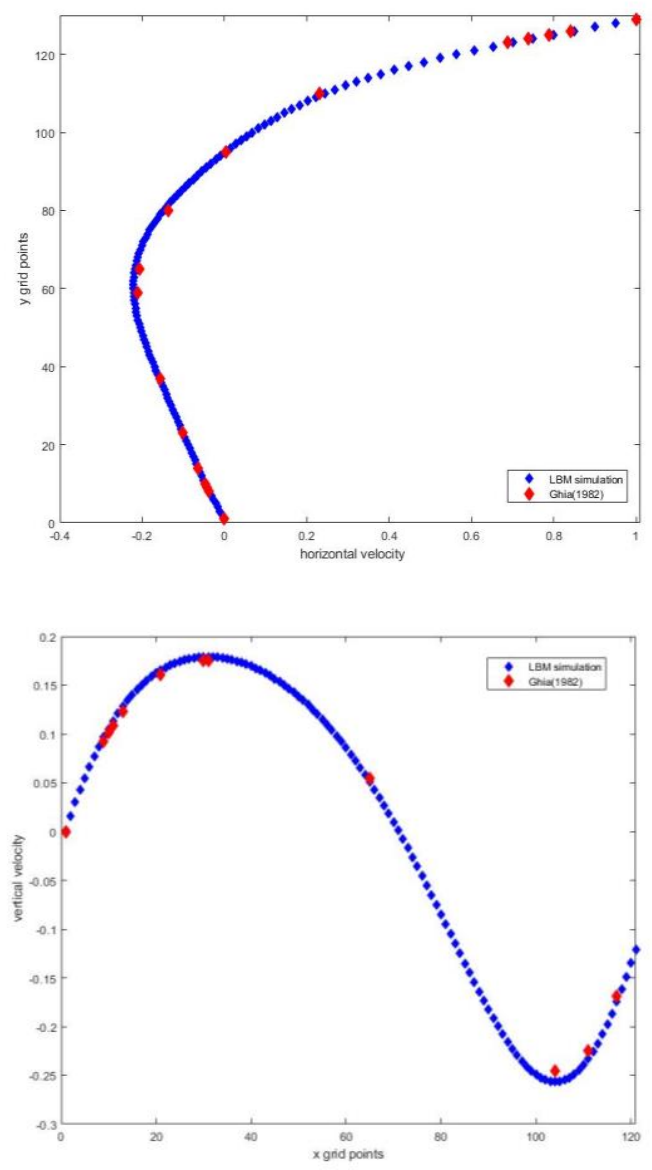

Figure 2: Plot of Horizontal and Vertical velocity with grid points for $\mathrm{Re}=100$ 
International Journal of Engineering Applied Sciences and Technology, 2019

Vol. 4, Issue 8, ISSN No. 2455-2143, Pages 14-26

Published Online December 2019 in IJEAST (http://www.ijeast.com)
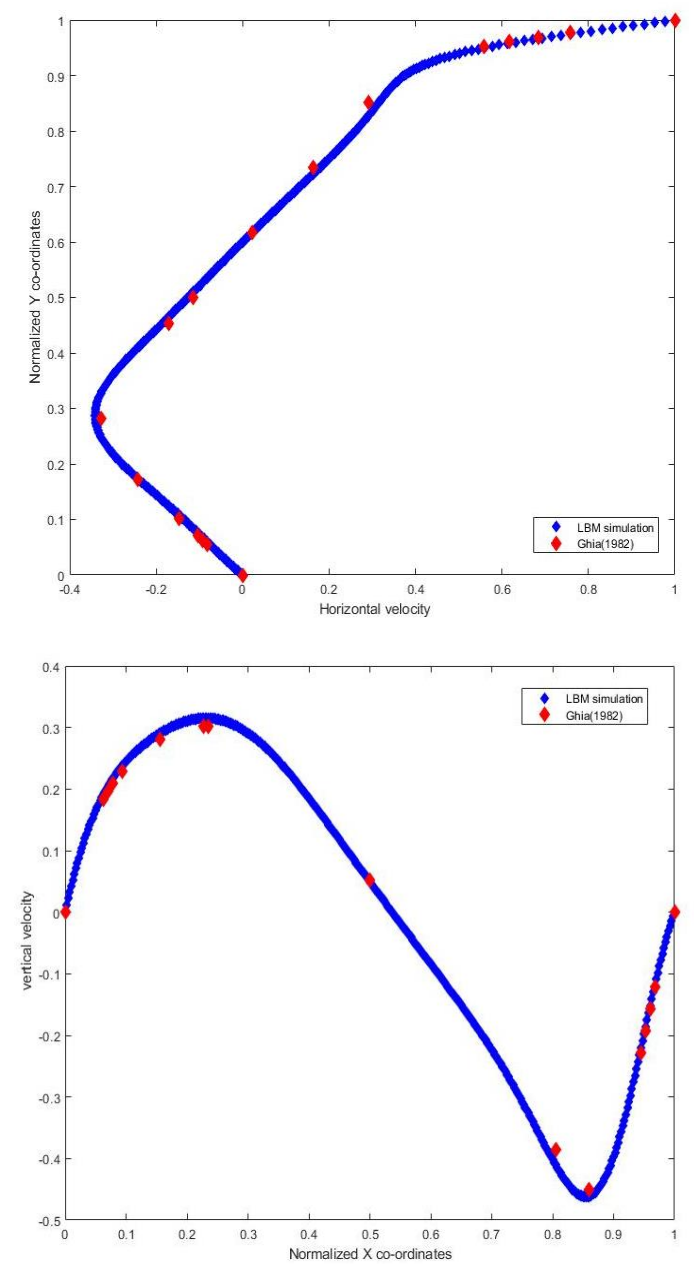

Figure 3: Plot of Horizontal and Vertical velocity with normalized co-ordinates for $\mathrm{Re}=400$

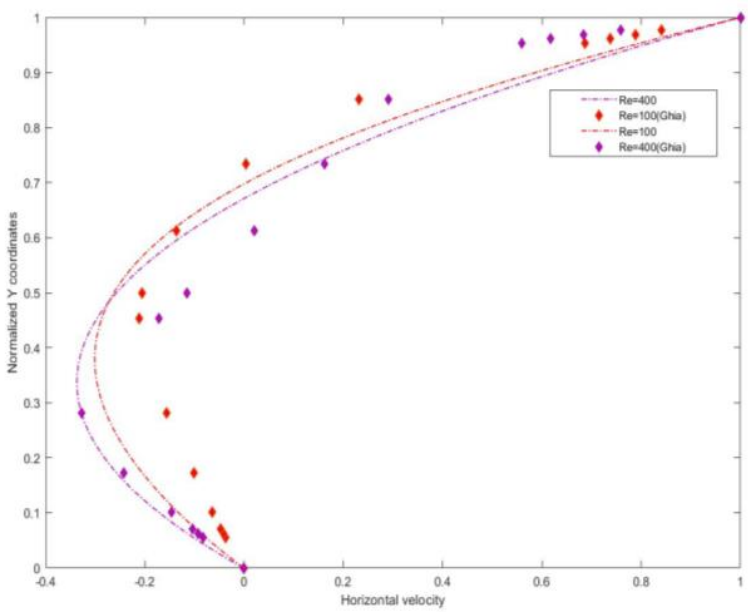

Figure 4: Plot of Horizontal velocity for $\mathrm{Re}=100$ and 400 for cavity with $\mathrm{Ar}=4$
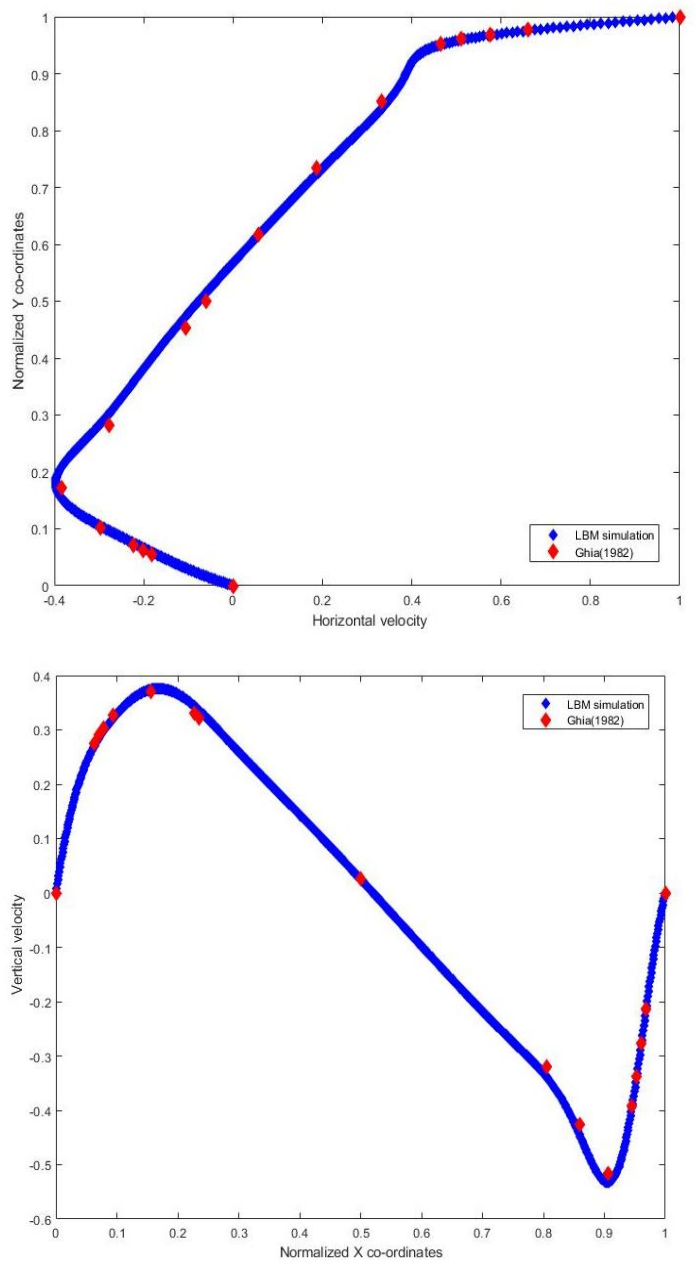

Figure 5: Plot of Horizontal and Vertical velocity with normalized co-ordinates for $\mathrm{Re}=1000$ 
International Journal of Engineering Applied Sciences and Technology, 2019 Vol. 4, Issue 8, ISSN No. 2455-2143, Pages 14-26

Published Online December 2019 in IJEAST (http://www.ijeast.com)
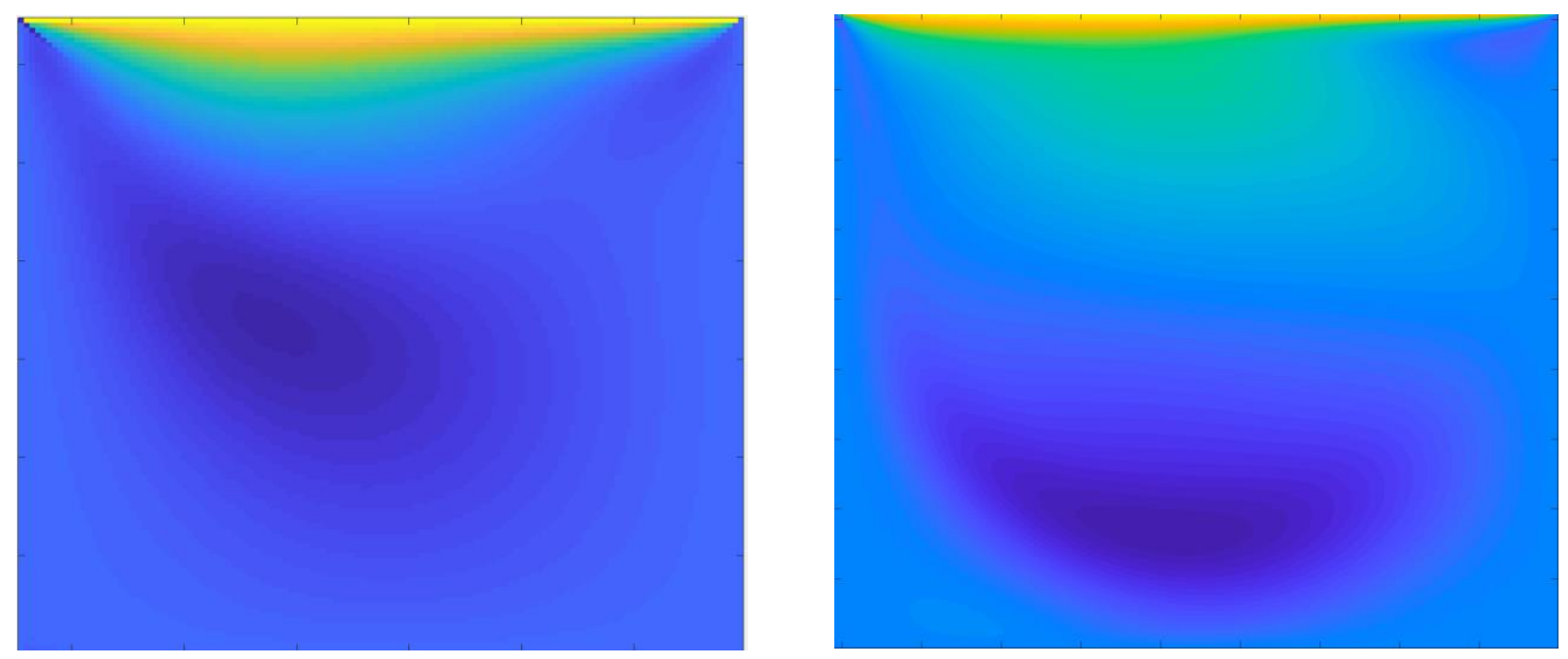

For $\mathrm{Re}=100$ (left) and 400(right) at $\mathrm{Ar}=1$
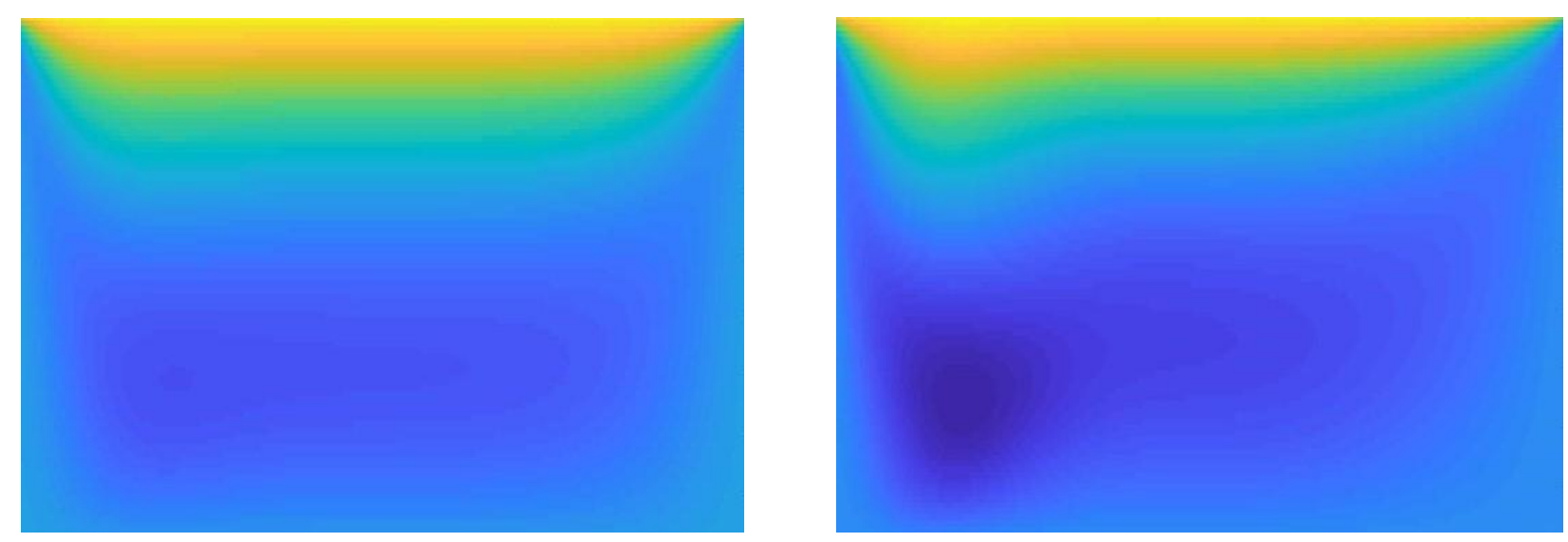

For $\mathrm{Re}=100$ (left) and 400(right) at $\mathrm{Ar}=4$

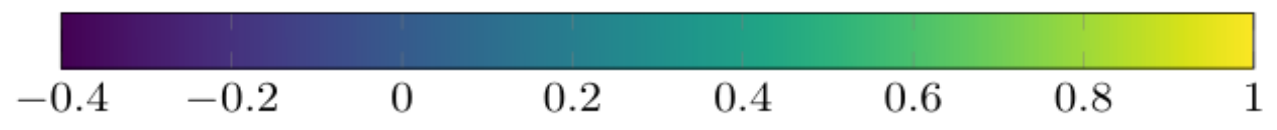

Figure 6: Contour of Horizontal Velocity 


\section{International Journal of Engineering Applied Sciences and Technology, 2019 \\ Vol. 4, Issue 8, ISSN No. 2455-2143, Pages 14-26 \\ Published Online December 2019 in IJEAST (http://www.ijeast.com)}

\section{THE RAYLEIGH BENARD PROBLEM}

The Rayleigh Benard system in which the fluid is confined between two parallel plates maintained with a temperature difference is one of the most studied non-equilibrium hydrodynamic systems.The geometrical setup for the Rayleigh problem involves two parallel plates separated by a distance $\mathrm{H}$. One plate is kept at a higher temperature than the other.Here we have discussed for both the horizontal and vertical test cases Fig. 7 ad Fig. 8.The physics of the Rayleigh-Benard convection is governed by the Rayleigh Number

$R a=\frac{g \beta\left(T_{h}-T_{c}\right) H^{3}}{\alpha v}$

which is the ratio of the temperature driven buoyancy force and viscous friction forces. $\alpha$ and $v$ are the thermal diffusivity and kinematic viscosity of the fluid respectively.Prandtl number $\operatorname{Pr}=\frac{v}{\alpha}$ also plays an important role in this phenomenon. The Boussinesq approximation states that the effect of a small density change creates a buoyancy force density

$$
F_{b}=-\beta \rho_{0}\left(T-T_{0}\right) g
$$

in the presence of a gravitational field with acceleration g.The advantage of Boussinesq approximation is that the temperature effects only enters through the body force density.After absorbing the force term the thermo-hydrodynamic equation is given by

$\frac{\partial u}{\partial t}+\Delta .(u u)=-\Delta p+v \Delta^{2} u-g \beta\left(T-T_{0}\right)$

$$
\frac{\partial T}{\partial t}+\Delta \cdot(u T)=\alpha \Delta^{2} T
$$

where $\mathrm{u}$ and $\mathrm{T}$ denotes the velocity and temperature field.The parameters $v, \kappa, \beta$ are kinematic viscosity , thermal diffusivity and thermal expansion coefficient.For implementing the lattice boltzmann method eq(15) and eq(16) are taken into consederation.

\subsection{Numerical Methodology:Two Polpulation Thermal Lattice Boltzmann Model}

The Thermal Lattice Boltzmann model involves two steps- streaming and collision of two distribution functions. We have taken ' $f$ ' as the mass density distribution and ' $g$ ' as the temperature distribution with D2Q9 and D2Q5 model respectively.The two distributions obey their respective lattice boltzmann transport equation with SRT BGK approximation.The streaming and collision of each of the distribution function is given by

$$
f_{i}\left(x+e_{i} \Delta t+t+\Delta t\right)-f_{i}(x, t)=\frac{\Delta t}{\tau_{f}}\left[f_{i}^{e q}(x, t)-\right.
$$$$
\left.f_{i}(x, t)\right]+F_{i}
$$

$g_{i}\left(x+e_{i} \Delta t+t+\Delta t\right)-g_{i}(x, t)=\frac{\Delta t}{\tau_{g}}\left[g_{i}^{e q}(x, t)-\right.$ $\left.g_{i}(x, t)\right]$

where $F_{i}$ is the force due to Boussinesq approximation. The relaxation times of both the distributions are given by the relations $v=c_{s}^{2}\left(\tau_{f}-\right.$ $1 / 2)$ and $\alpha=c_{s}^{2}\left(\tau_{g}-1 / 2\right)$ where $c_{s}$ is the lattice speed of sound.For each of the distributions the equilibrium distribution are given by

$$
\begin{aligned}
& f_{i}^{e q}(x, t)=w_{i} \rho\left(1+3 \frac{e_{i} u}{c}+\frac{\left(e_{i} u\right)^{2}}{c^{2}}-\frac{u \cdot u}{c^{2}}\right) \\
& g_{i}^{e q}=w_{g} T\left(1+3 \frac{e_{i} u}{c}\right)
\end{aligned}
$$

The introduction of force $F_{i}$ in eq(17) is given by $F_{i}(x, t)=3 w_{i} g \beta\left[T(x, t)-T_{0}\right] \rho(x, t) e_{i y} \quad$ following the forcing scheme with Boussinesq approximation. The flow macroscopic properties are defined by $\rho=\Sigma_{i} f_{i}, \rho u=\Sigma_{i} f_{i} e_{i}$ and $T=\Sigma_{i} g_{i}$ for density,moment flux and temperature respectively.The characteristic velocity scale $V \equiv$ $\sqrt{\beta g_{y} \Delta T H}$ is equal to $M a c_{s}$.The subsequent parameters are calculated by

$$
\begin{aligned}
& v^{2}=\frac{V^{2} l^{2} P r}{R a} \\
& \alpha=\frac{v}{P r}
\end{aligned}
$$

The average Nusselt Number of the flow is given by

$$
\bar{N} u=\frac{l}{\Delta T} \frac{1}{L H} \iint_{S} q(x, y) d x d y
$$

where $q(x, y)=u T-\alpha \frac{\partial T}{\partial x}$ is the local flux and L and $\mathrm{H}$ are the length and height of the domain.The $l$ denotes of the length scale specific to the problem.

\subsection{CASE:Temperature difference across the} Horizontal Boundary

The geometric setup of the first test case is given in the Fig. 7.A temperature difference, $\Delta T=$ $T_{h}-T_{c}$ is applied across the horizontal walls of the domain. The gravity is acting in the vertical direction with adiabatic walls on the top and bottom.

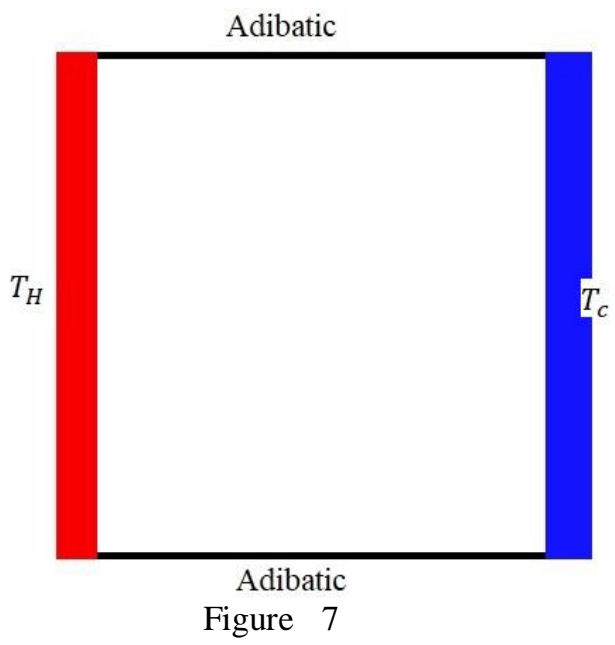


International Journal of Engineering Applied Sciences and Technology, 2019

Vol. 4, Issue 8, ISSN No. 2455-2143, Pages 14-26

Published Online December 2019 in IJEAST (http://www.ijeast.com)

Table 1: The value of the $\mathrm{N}^{-} \mathrm{u}$ for different grid domain with the benchmark solution

\begin{tabular}{|c|c|c|c|c|}
\hline Rayleigh Number & Grid & Benchmark(DeVahl Davis) & LBM & Error \\
\hline 1000 & & 1.118 & & \\
\hline & $181 \times 181$ & & 1.218 & $0.33 \%$ \\
\hline & $250 \times 250$ & & 1.1197 & $0.15 \%$ \\
\hline & $300 \times 300$ & & 1.1186 & $0.05 \%$ \\
\hline 10000 & & & 2.2248 & \\
\hline & $181 \times 181$ & & 2.235 & $0.81 \%$ \\
\hline & $250 \times 250$ & & 2.25 & $0.35 \%$ \\
\hline & $300 \times 300$ & & & \\
\hline & & & 4.4503 & $1.81 \%$ \\
\hline & $181 \times 181$ & & 4.4735 & $1.0 \%$ \\
\hline & $250 \times 250$ & & & $0.86 \%$ \\
\hline & $300 \times 300$ & & 8.335 & $5.5 \%$ \\
\hline & & & 8.71 & $1.3 \%$ \\
\hline & $181 \times 181$ & & & $0.59 \%$ \\
\hline & $250 \times 250$ & & & \\
\hline
\end{tabular}

In the simulation, the $2 \mathrm{D}$ cavity is mapped with square lattice in which $\Delta x=\Delta y$ with $\Delta t=1$. Grid independence study is performed for $\mathrm{Ra}=10^{3}, 10^{4}, 10^{5}$ and $10^{6}$ with $\operatorname{Pr}=0.71$. The average Nusselt number given in eq.(21) is calculated for each of the cases and compared with the existing benchmark solution of DeVahl Davis deVahlDavis et al (1983)] to check for correctness of the method. For a greater level of grid convergence the steady state criteria used is given by

$\max _{i, j}\left|\frac{T_{n}-T_{n-1}}{T_{n-1}}\right| \leq 10^{-7}$

is imposed. Considering the thermal domain, the left and right boundary are provided with a fixed temperature with the left at $T_{h}$ higher than the right $T_{c}$.For the temperature fixed boundaries, Dirichlet $\mathrm{BCs}$ are employed gived by

$$
\begin{gathered}
g_{1}=T_{h}-g_{0}-g_{2}-g_{3}-g_{4}: \text { leftoudary } \\
g_{3}=T_{c}-g_{0}-g_{1}-g_{2}-g_{4}: \text { rightboundary }
\end{gathered}
$$

For the top and bottom walls adiabatic conditions are employed through second order finite difference given by

$$
\begin{aligned}
& 3 T_{n}-4 T_{n-1}+T_{n-2}=0 \\
& -T_{1+2}+4 T_{1+1}-3 T_{1}=0
\end{aligned}
$$

The temperature at the boundary nodes calculated

from eq(24) is used to calculate for the distribution $g_{i}$. The problem can also be investigated through constant flux at the boundaries using Neuman BCs but we have limited our discussions to Dirichlet BCs only.

\subsubsection{Results}

The tabulated data Table. 1 is obtained after the stability criteria of is statisfied.In addition we investigated the isotherms and streamlines given in Fig. 9 to get a overall idea of the randomness of the system with the increase in Rayleigh Number. 
International Journal of Engineering Applied Sciences and Technology, 2019 Vol. 4, Issue 8, ISSN No. 2455-2143, Pages 14-26

Published Online December 2019 in IJEAST (http://www.ijeast.com)
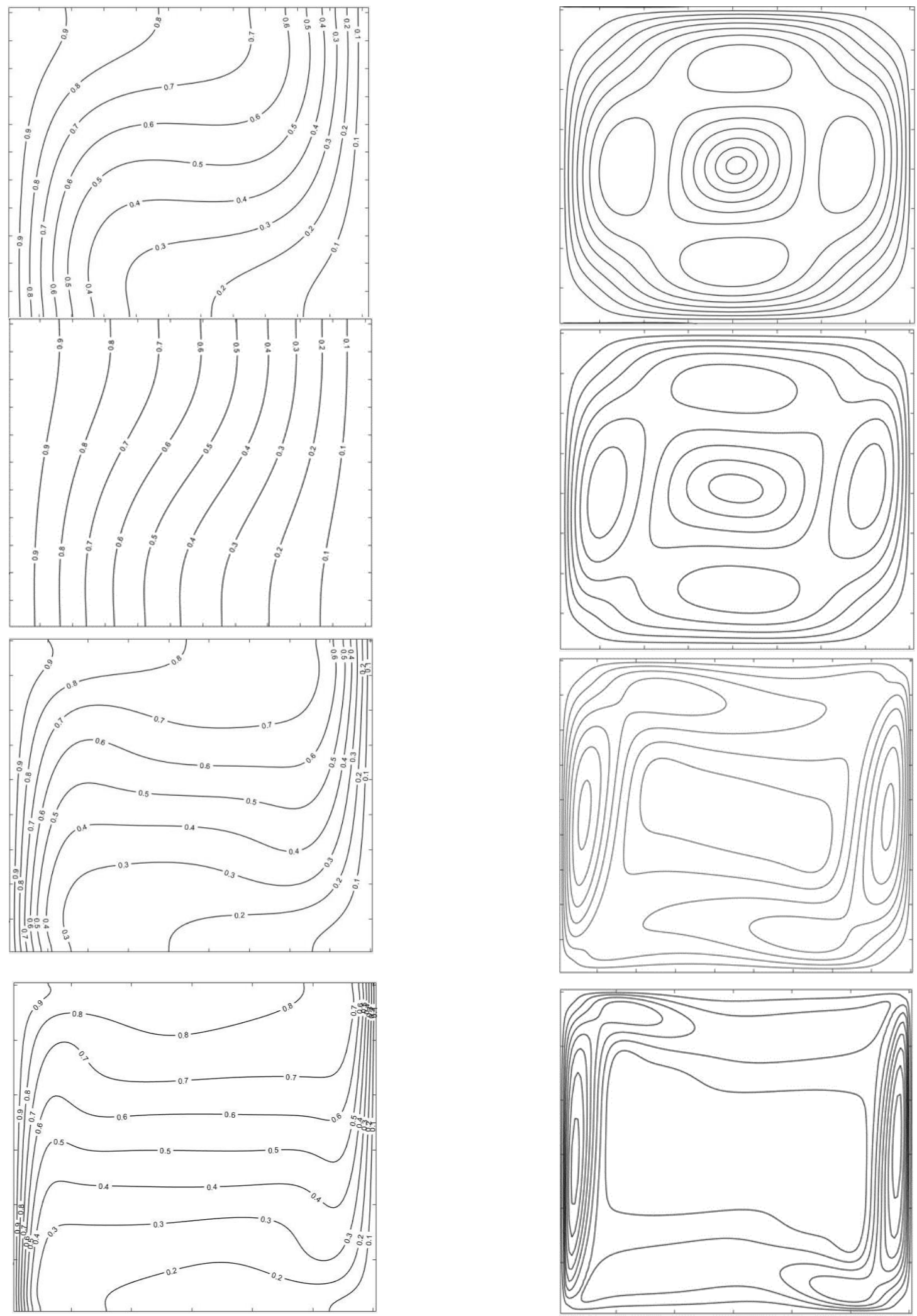

Figure 9: Isotherms and Streamlines for $\mathrm{Ra}=10^{3}, 10^{4}, 10^{5}$ and $10^{6}$ (Top to bottom) 


\section{International Journal of Engineering Applied Sciences and Technology, 2019 \\ Vol. 4, Issue 8, ISSN No. 2455-2143, Pages 14-26 \\ Published Online December 2019 in IJEAST (http://www.ijeast.com)}

\subsection{CASE: Temperature difference across the Vertical Boundary}

The geometric setup of the second test case is given in the Fig. 8.The same temperature difference, $\Delta T=T_{h}-T_{c}$ is applied across the vertical walls of the domain opposite to the direction in which gravity is acting.In the simulation the $2 \mathrm{D}$ cavity is mapped with rectangular lattice with a aspect ratio of 2 in which $\Delta x=\Delta y$ with $\Delta t=1$. Analysis are done for $\mathrm{Ra}=2000,10000$ and 50000 with $\operatorname{Pr}=0.71$. The average Nusselt number is calculated for $\operatorname{Pr}=0.71$ and 7 of the cases and compared with the MRT LBM simulations of Wang [Wang et al.(2013)Wang, Wang, Lallemand, and Luo].The stability criteria of the previous case succeeds in giving convincing results for this case also.For the fixed temperature vertical boundary conditions the same Dirichilet scheme is applied and the other two walls are provided with a periodic BCs.

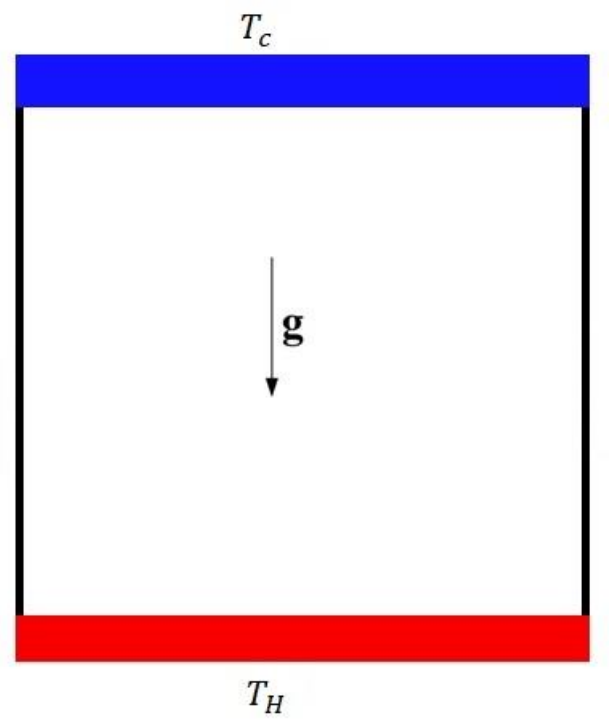

\subsubsection{Results}

We calculated the value of the $\bar{N} u$ to have a comparative study with the LBM (MRT) model of Wang et al.(2013).The isotherms for the different Rayleigh number is given in Fig.11.

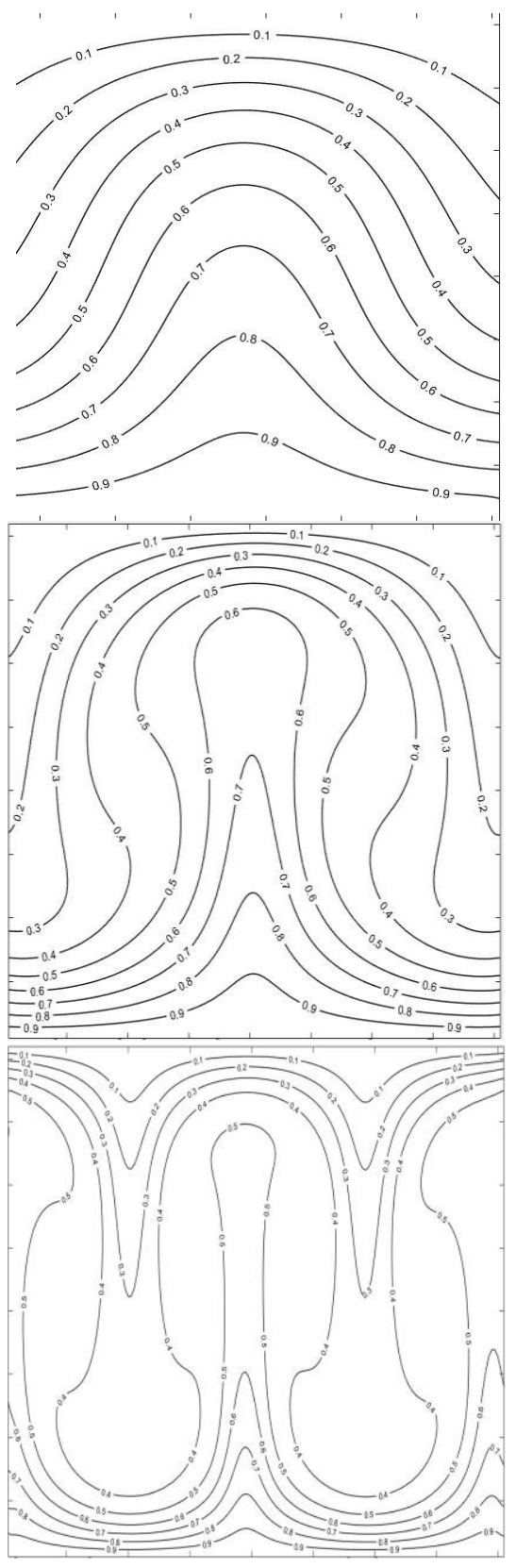

Figure 11: Isotherms of $\mathrm{Ra}=2000,10000$ and 50000

$\operatorname{Pr}=0.71 R a=10^{4}$

\begin{tabular}{|c|c|c|}
\hline Grid & LBM(SRT) & LBM(MRT) \\
\hline $82 \times 42$ & 2.5925 & 2.6621 \\
\hline $162 \times 82$ & 2.6571 & 2.656 \\
\hline
\end{tabular}

$\operatorname{Pr}=7 \quad R a=10^{4}$

\begin{tabular}{|c|c|c|}
\hline Grid & LBM(SRT) & LBM(MRT) \\
\hline $82 \times 42$ & 2.6228 & 2.6117 \\
\hline $162 \times 82$ & 2.716 & 2.6115 \\
\hline
\end{tabular}




\section{International Journal of Engineering Applied Sciences and Technology, 2019 Vol. 4, Issue 8, ISSN No. 2455-2143, Pages 14-26 \\ Published Online December 2019 in IJEAST (http://www.ijeast.com)}

\section{STEFAN PROBLEM OF PHASE TRANSITION}

An extended lattice boltzamnn method was developed for the simulation of the phase change problem governed by the heat conduction equation incorporated with the enthalpy formulation [Jiaung et al.(2001).In the enthalpy formulation approach the governing equation for the heat conduction problem expressed in terms of enthalpy is given by

$\frac{\partial(\rho H)}{\partial t}=\Delta \cdot(\lambda \Delta T)$

where total enthalpy, $H$ is divided into sensible enthalpy and latent heat for the phase change.

$$
H=C T+f_{l} L
$$

where $C$ and $L$ represents teh constant pressure specific heat and latent heat for the phase change and $f_{l}$ is the volume fraction of liquid. Substituting in eq(25) we get

$\frac{\partial(\rho C T)}{\partial t}=\Delta \cdot(\lambda \Delta T)-L \frac{\partial \rho f_{l}}{\partial t}$

where $\lambda$ is the thermal conductivity. The latent heat $\mathrm{L}$ appears in the source term.In dimensionless form

$\frac{\partial \theta}{\partial t}=\Delta^{2} \theta-\frac{1}{S t} \frac{\partial f_{l}}{\partial t}$

where $S t$ is the stefan number and $\theta$ is the non dimensional temperature. The enthalpy $H$ is used to calculate for the liquid fraction given by

$$
f_{l}=\frac{H-H_{S}}{H_{l}-H_{S}}
$$

Where $H_{s}$ and $H_{l}$ are the enthalpy values for the soild and liquid phase.The effective thermal difussivity can be represented as

$$
\alpha=\frac{\left(1-f_{l}\right) \lambda_{s}+f_{l} \lambda_{l}}{\left(1-f_{l}\right)(\rho C)_{s}+f_{l}(\rho C)_{l}}
$$

where $\lambda$ is the thermal conductivity.

The momentum equation is given by

$$
\frac{\partial u}{\partial t}+\Delta .(u u)=-\Delta p+v \Delta^{2} u+F
$$

where the force $F$ is considered to be zero for conduction case.

\subsection{Numerical Methodology:Enthalpy based Lattice Boltzmann model with Phase change}

We adopted two different model for temperature ad mass density distributions for the give problem.Density field is simulated through the D2Q9 model with 9 discrete directional distributions. For this the Lattice Boltzmann equation for streaming and collision is given by eq(5). For the temperature field D2Q5 model with 5 discrete directional distribution is taken into consideration. $g_{i}\left(x+c e_{i} \Delta t, t+\Delta t\right)-g(x, t)=-\frac{g_{i}(x, t)-g_{i}^{e q}(x, t)}{\tau_{g}}+$ $\Delta t \Phi$

where $\Phi$ corresponds to the source term introduced due to the phase change. The equilibrium distribution for D2Q5 model is

$g_{i}^{e q}=w_{g} T\left(1+3 \frac{e_{i} u}{c}\right)$

The relaxation times of both the distributions are given by the relations $v=c_{s}^{2}\left(\tau_{f}-1 / 2\right)$ and $\alpha=c_{s}^{2}\left(\tau_{g}-\right.$ $1 / 2)$ where $c_{s}$ is the lattice speed of sound.For the solidification and melting process the latent heat required for the phase change at the interface act as a source term.For the present LBM model we have considered the source term explicitly in the collision process given by

$g_{i}\left(x+c e_{i} \Delta t, t+\Delta t\right)-g(x, t)=-\frac{g_{i}(x, t)-g_{i}^{e q}(x, t)}{\tau_{g}}-$

$w g_{i} \Delta t \Psi$

where $\Psi=\frac{L}{C} \frac{\partial f_{l}}{\partial t}$. The same enthalpy based approach is applied to both solidification and melting process with different initial parameters.During the marching time step the iterative procedure is given by

1.The collision term at the new time level $t+$ $\Delta t$ is given by

$$
\begin{gathered}
g_{i}^{(n+1)}\left(x+c e_{i} \Delta t, t+\Delta t\right)=g_{i}^{n}(x, t)+ \\
\frac{g_{i}^{n}(x, t)-g_{i}^{e q}(x, t)}{\tau_{g}}-\Delta t w g_{i} \frac{L}{C} \frac{f_{l}^{n}(t)-f_{l}^{n-1}(t-\Delta t)}{\Delta t}
\end{gathered}
$$

where $n+1, n$ and $n-1$ are three consecutive time steps.

2.Then temperature is calculated by $T^{n+1}=$ $\Sigma_{i} g$.

3.The total enthalpy is updated by $H^{n+1}=$ $C T^{n+1}+f_{l}^{n} L$ where $C=\left(1-f_{l}^{n}\right) C_{s}+f_{l} C_{l}$ is the equivalent specific heat.

4.The liquid fraction of then updated

$$
f_{l}^{n+1}=\left(\begin{array}{ll}
0 & H^{n+1}<H_{s} \\
\frac{H^{n+1}-H_{S}}{H_{l}-H_{S}} & H_{S} \leq H^{n+1} \geq H_{l} \\
1 & H^{n+1}>H_{l}
\end{array}\right.
$$

The steps are iterated at each time step.

\subsection{CASE: One Region Melting Process}

The problem is defined as a one region melting problem subjected to a prescribed temperature.Initially the system is full solid at phase change temperature, $T_{m}$.At $\mathrm{t}=0$, the temperature at $\mathrm{x}=0$ i.e left boundary is raised to $T_{h}>T_{m}$ and maintained at that temperature for all times $t>0$ shown in Fig. 12.The problem is one region because the solid phase is at a constant temperature throughout and only unknown in the liquid phase.For the prescribed temperature case ,the solution was determined analytically given by 


\section{International Journal of Engineering Applied Sciences and Technology, 2019 \\ Vol. 4, Issue 8, ISSN No. 2455-2143, Pages 14-26 \\ Published Online December 2019 in IJEAST (http://www.ijeast.com)}

$T(x, t)=$

$\left(\begin{array}{ll}T_{h}-\left(T_{h}-T_{m}\right) \frac{\operatorname{erf}(x / 2 \sqrt{\alpha t})}{\operatorname{erf}(\lambda)} & x<X(t) \\ T_{m} & x>X(t)\end{array}\right.$

where $X(t)=2 \lambda \sqrt{\alpha_{l} t}$ is the position of the solid liquid interface. The parameter $\lambda$ is the solution of the transcendental equation given by

$\lambda e^{\lambda^{2}} \operatorname{erfc}(\lambda)=\frac{S t}{\sqrt{\pi}}$

where $\mathrm{St}=\rho C_{S} \frac{\left(T_{h}-T_{m}\right)}{L}$ is the Stefan number.The equivalent thermal diffusivity is given by the expression

$\alpha_{m}=\frac{\left(1-f_{l}\right) \lambda_{S}+f_{l} \lambda_{l}}{\left(1-f_{l}\right) \rho C_{S}+f_{l} \rho C_{l}}$

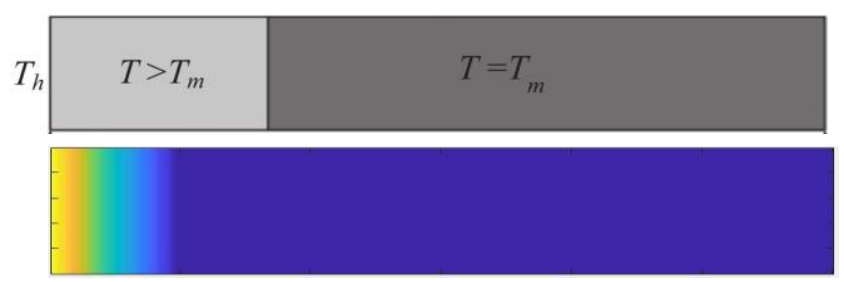

Figure 12: Schematic and Simulation representation of the one region melting problem

\subsubsection{Results}

The Stefan number is fixed at 0.125 with ratio of thermal conductivity $\frac{\lambda_{l}}{\lambda_{s}}=0.25$ and ratio of specific heat $\frac{C_{l}}{C_{s}}=4$. The analytical solution is plotted with the simulated results for each time for validation with the theoretical approach given in Fig. 13.

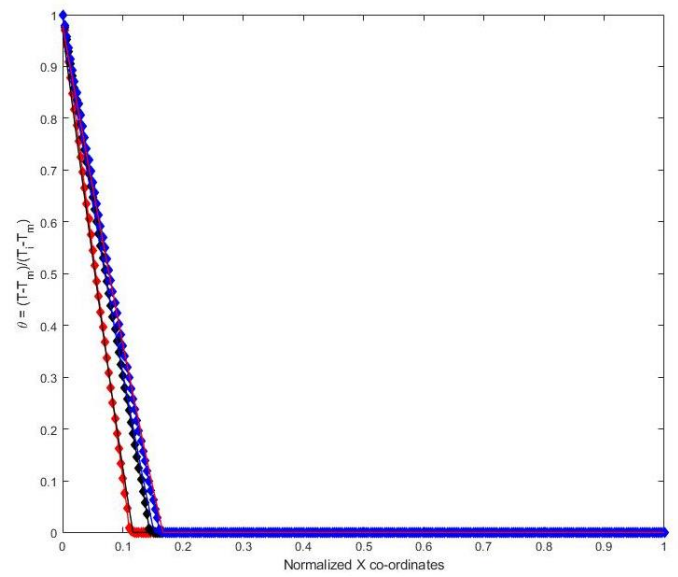

Figure 13: Plot of non-dimensional temperature $\left(\frac{T-T_{m}}{T_{h}-T_{m}}\right)$ at $2000 \mathrm{~s}, 6000 \mathrm{~s}$ and $11000 \mathrm{~s}$. The solid line represents the analytical solution

\subsection{CASE: Two Region Melting Process}

Initially, $\mathrm{t}=0$ the whole domain is in solid phase at temperature $T_{0}$ lower than the phase change temperature $T_{m}$. The temperature at the left boundary is suddenly raised to $T_{h}>$ with $T_{h}>T_{m}>T_{0}$ and maintained at all times $t>0$. Melting begins at the surface at $\mathrm{x}=0$ and proceed into the material.Fig. 14 represents the schematic representation of the problem. The temperature distribution can be solved analytically given by

$T(x, t)=$
$\left(\begin{array}{ll}T_{h}-\left(T_{h}-T_{m}\right) \frac{\operatorname{erf}(x / 2 \sqrt{\alpha t})}{\operatorname{erf}(\lambda)} & x \leq X(t) \\ T_{0}+\left(T_{m}-T_{0}\right) \frac{\operatorname{erfc}\left(x / 2 \sqrt{\alpha_{s} t}\right)}{\operatorname{erfc}\left(\lambda \sqrt{\alpha_{s} / \alpha_{l}}\right.} & x>X(t)\end{array}\right.$

where $\alpha_{s}=\frac{\lambda_{s}}{\rho C_{s}}$ and $\alpha_{l}=\frac{\lambda_{l}}{\rho C_{l}}$ are the thermal diffusivity of solid and liquid phase. The constant $\lambda$ is the solution of the analytical solution

$$
\frac{C_{l}\left(T_{h}-T_{m}\right)}{e^{\lambda^{2}} \operatorname{erf}(\lambda) L}-\frac{C_{S}\left(T_{m}-T_{0}\right) \sqrt{\alpha_{s} / \alpha_{l}}}{e^{\lambda^{2} \alpha_{l} / \alpha_{S}} \operatorname{erfc}\left(\lambda / \sqrt{\alpha_{s} / \alpha_{s}}\right) L}=\lambda \sqrt{\pi}
$$

The location of the interface of phase chage is given by $X(t)=2 \lambda \sqrt{\alpha_{l} t}$.

\section{$T>T_{n}$}

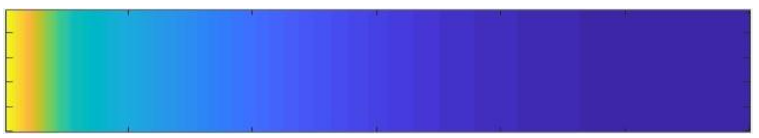

Figure 14: Schematic and Simulation representation of the two region melting problem

\subsubsection{Results}

The Stefan number is fixed at 0.25 with ratio of thermal conductivity $\frac{\lambda_{l}}{\lambda_{s}}=0.25$ and ratio of specific heat $\frac{C_{l}}{C_{s}}=2$.. The analytical solution is plotted with the simulated results for each time for validation with the theoretical approach given in Fig. 15.There is change in the profile for two different phase seperated by the interface at melting temperature. 
International Journal of Engineering Applied Sciences and Technology, 2019

Vol. 4, Issue 8, ISSN No. 2455-2143, Pages 14-26

Published Online December 2019 in IJEAST (http://www.ijeast.com)

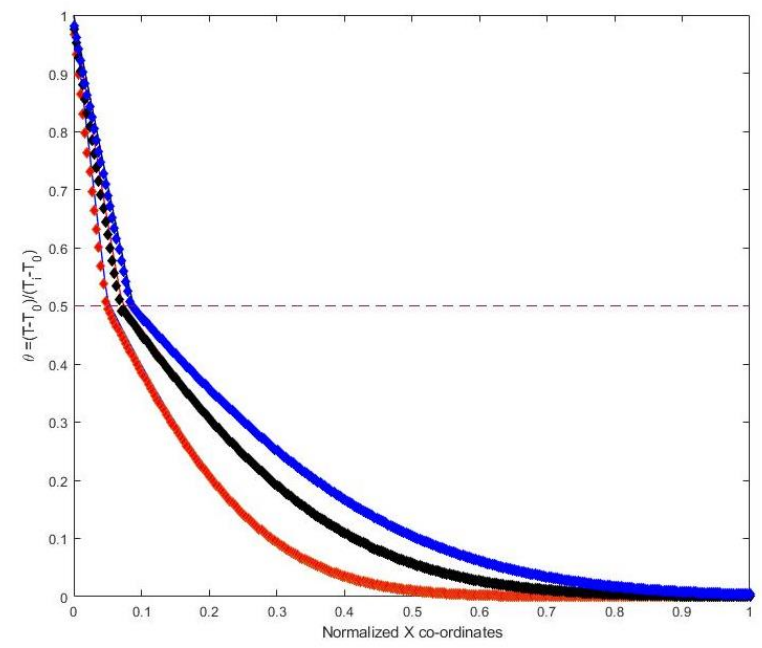

Figure 15: Plot of non-dimensional temperature $\left(\frac{T-T_{m}}{T_{h}-T_{m}}\right)$ at $2000 \mathrm{~s}, 6000 \mathrm{~s}$ and $11000 \mathrm{~s}$. The solid line represents the analytical solution. The dotted line represent the melting point

\subsection{CASE: Melting Process by convection}

Initially the substance is uniformly solid at melting temperature $T_{m}$. At $\mathrm{t}=0$, the temperature at left boundary is raised to a temperature $T_{h}>T_{m}$ while the other boundaries are assumed to be adiabatic.The simulation is carried out with following assumptions:(1)the fluid in the domain is incompressilble(2)Boussinesq approximation is applicable for the fluid.The problem is dependent of the following on-dimensional parameters : $R a=$ $\frac{g \beta \Delta T H^{3}}{v \alpha}, \operatorname{Pr}=\frac{v}{\alpha}, S t=C_{p} \frac{\Delta T}{L} \quad$ where $\quad \Delta T \quad$ is the temperature difference and the parameters are Rayleigh number,Prandtl number and Stefan number respectively.We focused on calculating Nusselt number at the left boundary for three different $R a=$ $2.5 \times 10^{3}, 2.5 \times 10^{4}$ and $2.5 \times 10^{5}$.

$N u_{h}=-\frac{L}{\Delta T} \int_{0}^{H} \frac{\partial T}{\partial x} d y$

where $\mathrm{L}$ is the length scale along the temperature difference.With the increase in Fourier number the Nusselt number is attaining a steady value given in Fig. 17.

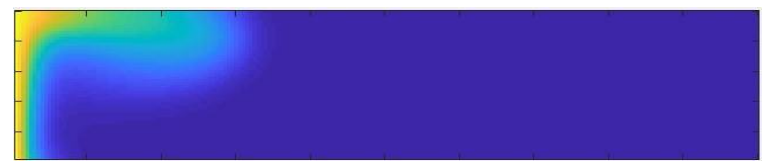

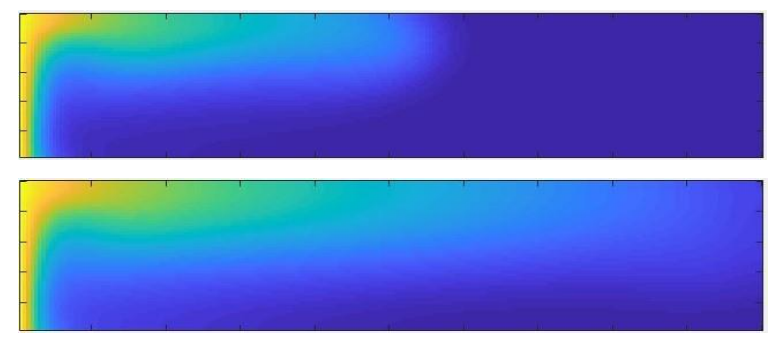

Figure 16: Isotherms for convection melting for consecutive time steps

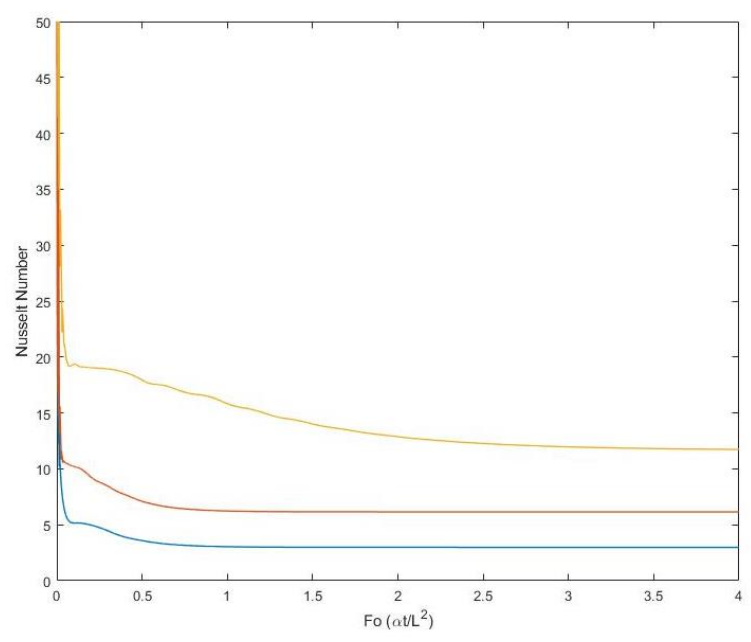

Figure 17: Nusselt numer at the left boundary with fourier number(Fo)

\section{CONCLUSION}

The lattice boltzmann model provides an efficient and effective way to simulate fluid flow problem with complex boundary conditions.In this study we investigated three different cases - the lid driven cavity flow,the rayleigh benard problem and the stefan problem of solidification and melting for different dimensionless parameters like reynolds number, rayleigh number, prandtl number,stefan number and provided excellent results in accordance to the existing benchmark solution.All the cases simulated are within the incompressible limit $(\mathrm{Ma}<0.3)$ with appropriate velocity scale.

\section{ACKNOWLEDGEMENTS}

This work was supported by the Mitacs Globalink Internship Programme and Polytechnique Montreal,Montreal,Quebec,Canada. 


\section{International Journal of Engineering Applied Sciences and Technology, 2019 Vol. 4, Issue 8, ISSN No. 2455-2143, Pages 14-26 \\ Published Online December 2019 in IJEAST (http://www.ijeast.com)}

\begin{tabular}{|c|c|c|}
\hline \multicolumn{2}{|c|}{ NOMENCLATURE } & \\
\hline$\overline{f_{i}}$ & $\begin{array}{l}\text { Population } \\
\text { distribution }\end{array}$ & \\
\hline $\bar{\Omega}$ & Collision operator & \\
\hline$u, U$ & Velocity & \\
\hline$\rho$ & Density & $(\mathrm{m} / \mathrm{s})$ \\
\hline$v$ & kinematic viscosity & $\left(\mathrm{kg} / \mathrm{m}^{3}\right)$ \\
\hline$R e$ & Reynolds number & $\left(m^{2} / s\right)$ \\
\hline$R a$ & Rayleigh number & \\
\hline$T_{h}, T_{c}$ & Temperature & \\
\hline$\beta$ & $\begin{array}{l}\text { Thermal Expansion } \\
\text { Coefficient }\end{array}$ & $(\mathrm{K})$ \\
\hline$\alpha$ & Thermal Diffusivity & $\left(T^{-1}\right)$ \\
\hline$g_{i}$ & $\begin{array}{l}\text { Temperature } \\
\text { population } \\
\text { distribution }\end{array}$ & $\left(m^{2} / s\right)$ \\
\hline$g$ & Gravity & \\
\hline $\mathrm{Nu}$ & Nusselt Number & \\
\hline$q(x, y)$ & Heat flux & $\left(\mathrm{m} / \mathrm{s}^{2}\right)$ \\
\hline$H$ & Enthalpy & \\
\hline$C$ & Specific Heat & $\begin{array}{|ll|} & (\quad W / \\
\left.m^{2}\right) & \\
\end{array}$ \\
\hline$\overline{f_{l}}$ & $\begin{array}{l}\text { Liquid } \quad \text { phase } \\
\text { fraction }\end{array}$ & (J) \\
\hline$L$ & Latent heat & (J/(K.kg \\
\hline$\theta$ & $\begin{array}{l}\text { Non dimensional } \\
\text { Temperature }\end{array}$ & \\
\hline$\lambda$ & $\begin{array}{l}\text { Thermal } \\
\text { conductivity }\end{array}$ & $(\mathrm{J} / \mathrm{kg})$ \\
\hline$S t$ & Stefan number & \\
\hline
\end{tabular}

\section{REFERENCES}

[1] Bennett, S., A lattice Boltzmann model for diffusion of binary gas mixtures, $\mathrm{PhD}$ thesis, University of Cambridge, (2010).

[2] Chakraborty, S. and Chatterjee, D., "An enthalpybased hybrid lattice-boltzmann method for modelling solid-liquid phase transition in the presence of convective transport," Journal of Fluid Mechanics, 592, pp. 155-175, (2007).
[3]Chatterjee, D., "Lattice boltzmann simulation of incompressible transport phenomena in macroscopic solidification processes," Numerical Heat Transfer, Part B: Fundamentals, 58(1), pp. 55-72, (2010).

[4] de Vahl Davis, G., "Natural convection of air in a square cavity: a bench mark numerical solution," International Journal for numerical methods in fluids, 3(3), pp. 249-264, (1983).

[5] Ghia, U., Ghia, K. N., and Shin, C., "High-re solutions for incompressible flow using the navierstokes equations and a multigrid method," Journal of computational physics, 48(3), pp. 387-411, (1982).

[6] Guo, Z., Shi, B., and Zheng, C., "A coupled lattice bgk model for the boussinesq equations," International Journal for Numerical Methods in Fluids, 39(4), pp. 325-342, (2002).

[7] Huang, R. and Wu, H., "Phase interface effects in the total enthalpy-based lattice boltzmann model for solid-liquid phase change," Journal of Computational Physics, 294, pp. 346-362, (2015).

[8] Huber, C., Parmigiani, A., Chopard, B., Manga, M., and Bachmann, O., "Lattice boltzmann model for melting with natural convection," International Journal of Heat and Fluid Flow, 29(5), pp. 14691480, (2008).

[9] Huo, Y. and Rao, Z., "The quasi-enthalpy based lattice boltzmann model for solid-liquid phase change," Applied Thermal Engineering, 115, pp. 1237-1244, (2017).

[10] Jiaung, W.-S., Ho, J.-R., and Kuo, C.-P., "Lattice boltzmann method for the heat conduction problem with phase change," Numerical Heat Transfer: Part B: Fundamentals, 39(2), pp. 167-187, (2001).

[11] Kao, P.-H. and Yang, R.-J., "Simulating oscillatory flows in rayleigh-benard convection using the lattice boltzmann method," International Journal of Heat and Mass Transfer, 50(17-18), pp. 3315-3328, (2007).

[12] Wang, J., Wang, D., Lallemand, P., and Luo, L.S., "Lattice boltzmann simulations of thermal convective flows in two dimensions," Computers \& Mathematics with Applications, 65(2), pp. 262-286, (2013). 